\title{
Assessing anterior commissure in rigid laryngoscopy
}

\author{
Al-Zahid S, Hickey S, Reece P \\ ENT department, Torbay Hospital, Devon, UK
}

\section{Background}

Involvement of the anterior commissure in early glottic laryngeal tumours has increased recurrence rate whether treated with laser excision ${ }^{1},{ }^{2}$ or radiotherapy ${ }^{3}$. Assessment of the anterior commissure with rigid laryngoscopy under a general anaesthetic is often difficult especially when confounded by prominent teeth, a large tongue, a receding jaw, and a high larynx. The presence of saliva or blood can also hinder the view. Laryngoscopes often have an anterior angle to facilitate viewing of the anterior commissure, and straight suction devices do not have the corresponding anterior angle needed. We hence devised a simple method to get around the bend to suction anteriorly with the help of a 30 degrees scope and assess this area appropriately.

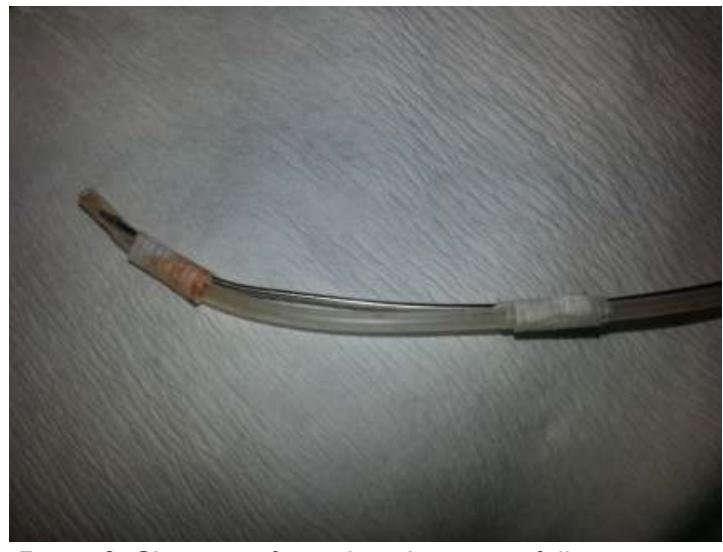

Figure 2. Close up of metal guidewire tip following removal from Hunsaker jet ventilation tube and attachment to suction tubing using steri-strips

\section{Technique}

For all micro-laryngeal surgery, we use a hunsaker tube to jet ventilate the patient who would be spontaneously breathing. The disposable hunsaker tube is bent to the angle of the laryngoscope blade to allow intubation of the patient. After removing the metal guide-wire, rather than disposing this, a suction catheter is attached with steri-strips, so that the suction catheter assumes a similar curve (Figure 1 and 2). This can then be attached to the suction connection and used to clear the secretions from the anterior commissure (Figure 3 ).

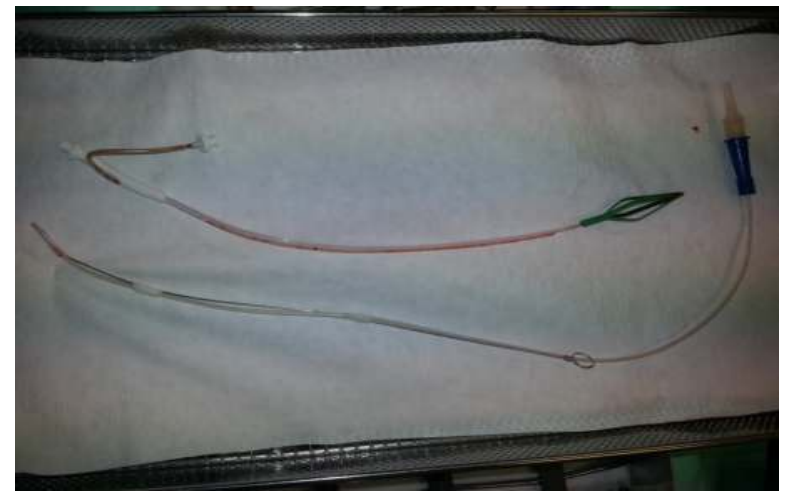

Figure I. Hunsaker jet ventilation tube with metal guidewire removed and attached to suction tubing using steri-strips

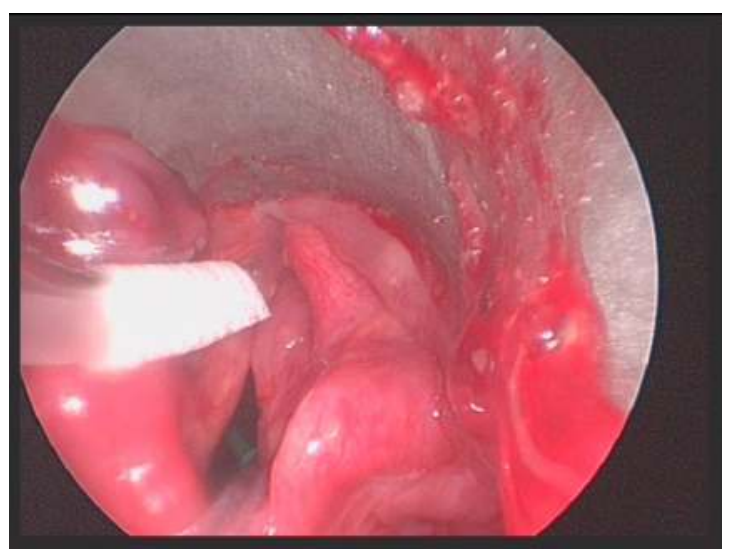

Figure 3. Close up of metal guidewire tip following removal from Hunsaker jet ventilation tube and attachment to suction tubing using steri-strips

\section{Discussion}

This technique can be seen as an addition to the armamentarium of the surgeon when faced with a difficult laryngoscopy procedure due to patient's body habitus.

\section{Bullet point summary}

- Anterior commissure assessment is vital for staging of laryngeal lesions

- When access is limited suction using a straight metal sucker is challenging

- A novel technique is described using a bendable curved sucker and a 30 degrees endoscope

\section{References}

I - Chone CT,Yonehara E, Martins JEF,Altemani A, Crespo AN. Importance of anterior commissure in recurrence of early glottic cancer after laser endoscopic resection Arch Otolaryngol Head Neck Surg 2007; I33(9): 882-887

2 - Rodel RMW, Steiner W, Muller RM, Kron M, Matthias C. Endoscopic laser surgery of early glottic cancer: involvement of the anterior commissure Head Neck 2009; 31 (5): 583-92

3 - Persky MS, Lagmay VM, Cooper J, et al. Curative radiotherapy for anterior commissure laryngeal carcinoma Ann Otol Rhinol Laryngol 2000; 109(2): 156-159 